\title{
COLAB-5: Um Modulo para Simulação de Redes 5G
}

\author{
Gabriel C. Ferreira ${ }^{1}$, Luis A. B. Pacheco ${ }^{1}$, Priscila Solís Barreto ${ }^{1}$, \\ Marcos F. Caetano ${ }^{1}$, Eduardo A. P. Alchieri ${ }^{1}$, Rodrigo Cavalcanti ${ }^{2}$ e Diego Aguiar Sousa ${ }^{2}$ \\ ${ }^{1}$ Departamento de Ciência da Computação - Universidade de Brasília (UnB) \\ Caixa Postal 4466 CEP 70910-900 - Brasília - DF - Brasil \\ \{luisbelem, gabrielcarvfer\}@aluno.unb.br, \{pris,mfcaetano, alchieri\}@unb.br
}

${ }^{2}$ GTEL, Departamento de Engenharia de Teleinformática Universidade Federal Do Ceará (UFC) Caixa Postal 6005-CEP 60440-900 - Fortaleza - CE - Brasil

\{rodrigo, diego\}@gtel.ufc.br

\begin{abstract}
This work presents COLAB-5, a tool developed for the NS-3 system simulator, which allows the simulation of different scenarios in $5 G$ networks. The tool was modeled by following a modular approach that allows the incorporation of physical layer characteristics in the simulator through a pre-defined interface and JSON technology. The results produced by a physical link level simulator - in this case $5 G$ for remote areas - are incorporated into the NS3 simulator. This approach allows the simulation of varied scenarios in NS3 and reduces the computational complexity by simulating different scenarios. COLAB-5 also implements CR (Cognitive Radios) techniques in the NS-3 MAC layer, based on collaborative sensing that allows the opportunistic use of the electromagnetic spectrum. In this article, the operation of COLAB-5 is illustrated in a $5 G$ environment for remote areas, which uses frequencies between $170 \mathrm{MHZ}$ to $700 \mathrm{MHz}$ and performs opportunistic access on TVWS (TV White Spaces) channels.
\end{abstract}

Resumo. Este trabalho apresenta o COLAB-5, uma ferramenta desenvolvida para o simulador de sistema NS-3, que permite a simulação de diversos cenários em redes 5G. A ferramenta foi modelada ao seguir uma abordagem modular que permite a incorporação de características da camada física no simulador mediante uma interface pré-definida e a tecnologia JSON. Os resultados produzidos por um simulador de nível de enlace físico - neste caso de $5 G$ para áreas remotas - são incorporados no simulador NS-3. Essa abordagem permite a simulação de cenários variados no NS-3 e reduz a complexidade computacional ao simular diversos cenários. O COLAB-5 também implementa técnicas de $R C$ (Radios Cognitivos) na camada MAC do NS-3, baseadas no sensoriamento colaborativo que permitem o uso oportunístico do espectro eletromagnético. Neste artigo, o funcionamento do COLAB-5 é ilustrado em um ambiente de $5 G$ para áreas remotas, que utiliza frequências entre $170 \mathrm{MHZ}$ a $700 \mathrm{MHz}$ e executa acesso oportunístico em canais de TVWS (TV White Spaces).

\section{Introdução}

A América Latina experimentou um crescimento dramático de assinantes de internet móvel nos últimos anos. De acordo com a GSMA [Association 2017], de todos os assinantes de internet móvel, mais de dois terços se conectam à internet via redes de banda 
larga móvel ( $3 \mathrm{G}$ ou $4 \mathrm{G}$ ). Apesar desta taxa de crescimento, somente $55 \%$ da população latino-americana (aproximadamente, 640 milhões de pessoas) possui assinatura de internet móvel. Em 2020, espera-se um crescimento no provimento deste serviço, estimandose o seu alcance a dois terços da população. Contudo, neste cenário, 250 milhões de pessoas devem permanecer excluídas digitalmente, em sua maioria vivendo em áreas remotas ou rurais e de baixa cobertura.

Uma das formas para alcançar a inclusão digital nas redes $5 \mathrm{G}$ é por meio da definição de uma tecnologia específica para áreas rurais ou remotas, em conjunto com um plano de negócios que seja atraente para as operadoras. Elementos base neste cenário são o baixo custo no licenciamento do espectro e seu uso eficiente, bem como a definição de uma camada física que opere em frequências especialmente voltadas para essas áreas e suas aplicações [Agnelli et al. 2014]. Outro fator importante a ser considerado, é a disponibilidade das bandas de TV analógica que ficaram sem uso após a transição do sistema analógico para o digital, conhecidas como TV Whitespaces (TVWS). Essas faixas de frequência podem ser utilizadas na implementação de técnicas de Radio Cognitivo (RC) para um melhor aproveitamento e uso eficiente do espectro disponível.

A tecnologia de RC é considerada fundamental para mitigar o problema de subutilização do espectro e otimizar a alocação de recursos. O espectro de radiofrequência apresenta um conjunto de faixas não contínuas e subutilizadas pelos usuários licenciados, conhecidos como usuários primários (PUs em inglês). O sensoriamento de espectro é o primeiro passo para o seu compartilhamento. Esta técnica permite a identificação, ao longo do tempo, das faixas subutilizadas e permite que usuários secundários (SUs em inglês) possam utilizá-as de maneira dinâmica e oportunística, liberando o seu uso tão logo o PU seja detectado. Este processo é conhecido como acesso dinâmico do espectro (DSA - Dynamic Spectrum Access) [Yau et al. 2018].

Amplamente usado no meio acadêmico, o NS-3 [NSNAM 2019] é um simulador de rede de eventos discretos de código aberto. $\mathrm{O}$ interesse em estender as funcionalidades do NS-3 para avaliar tecnologias e propostas em 5G, seja em áreas remotas e urbanas, pode permitir a avaliação de novos modelos de canal, técnicas de RC, DSA, entre outras. Alguns módulos disponíveis tem dado suporte à simulação de recentes tecnologias de redes móveis, por exemplo o LENA [CTTC 2019], desenvolvido para o LTE (Long Term Evolution). Até o presente momento, onde é de conhecimento destes autores, não foi encontrada nenhuma implementação sólida, de código aberto, que permita a simulação de uma rede $5 \mathrm{G}$ concomitantemente com novas tecnologias de camada física e de $\mathrm{RC}$ na camada de enlace.

Este trabalho apresenta o COLAB-5, que implementa um conjunto de adaptações nas camadas física e de enlace no módulo LTE do simulador NS-3, para viabilizar uma maior flexibilidade na alteração da sincronização dos protocolos e carregamento de novos modelos de canal, além de técnicas de sensoriamento colaborativo para acesso oportunístico do espectro. O COLAB-5 foi validado utilizando modelagem de canal e cenários de redes 5G em áreas remotas. A implementação e validação deste módulo para o NS-3 com a pilha LTE serve de base para a simulação de uma rede 3GPP (3rd Generation Partnership Project) e dessa forma, se posiciona junto às iniciativas de padronização do $5 \mathrm{G}$ [Sultan 2019]. As contribuições esperadas do COLAB-5 à área de pesquisa em redes são as seguintes: a) validação de novos modelos de canal e técnicas de modulação/codificação 


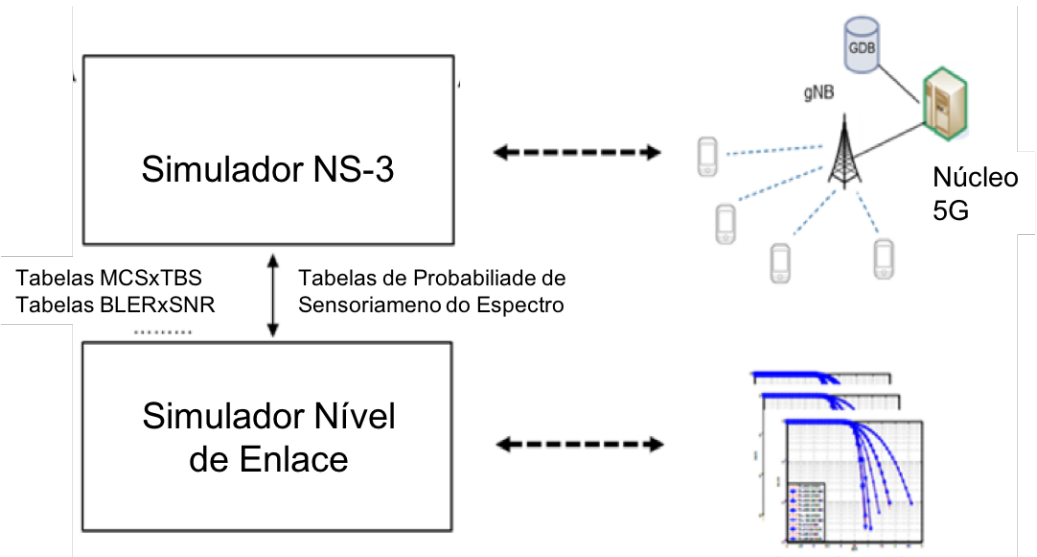

Figura 1. Abordagem da Implementação no NS-3.

da camada física, em cenários mais complexos que os permitidos em testes de campo; b) suporte ao desenvolvimento de novas técnicas de RC; c) avaliação de métricas das diversas aplicações de 5G em cenários diversos; d) disponibilizar uma ferramenta de código aberto que possa ter a colaboração de diversos grupos interessados na área.

Este artigo está organizado como segue: a Seção 2 apresenta a especificação da arquitetura usada no NS-3 para implementar as alterações na camada física e camada de enlace. A Seção 3 descreve o roteiro de demonstração da ferramenta em um caso de uso. A Seção 4 apresenta as conclusões e trabalhos futuros.

\section{Descrição da ferramenta}

\subsection{Alterações na Camada Física}

Nesta seção é descrita a implementação das novas funcionalidades no LENA, as quais seguem na direção das especificações 3GPP [Sultan 2019].

A Figura 1 ilustra a abordagem utilizada na integração entre o simulador de camada física e o simulador de sistema NS-3. O simulador no nível de enlace físico, implementado em MATLAB, fornece como resultado um conjunto de tabelas que descrevem e modelam o comportamento do canal em diversos cenários. Resultados de outros modelos de canal podem ser gerados por outras ferramentas e carregados seguindo o mesmo formato, adaptando o simulador as necessidades do usuário. Os valores fornecidos são: a relação sinal ruído e de interferência (SNR - Signal Noise Ratio), a eficiência espectral, a vazão alcançável utilizando um determinado esquema de modulação e codificação (MCS - Modulation and Coding Scheme), a taxa de erro por bloco (BLER - Block Error Rate) e o tamanho do bloco ou quadro (TBS -Transport Block Size), este último descrevendo a resiliência do MCS à interferência. No MATLAB também são produzidas as informações de sensoriamento do espectro, as quais são representadas por curvas de probabilidade de detecção dos usuários primários. Estas curvas são utilizadas, no NS-3, para a implementação do mecanismo de sensoriamento colaborativo. Todas as informações descritas são exportadas em formato JSON e carregadas pelo NS-3 durante a simulação de nível de sistema. Dessa maneira as propriedades da camada física são incorporadas ao simulador de sistema e integradas às camadas superiores, o que permite a simulação de cenários mais complexos. Esta abordagem reduz a complexidade computacional da 
simulação, ao separar o processo em duas etapas e permite uma maior flexibilidade na integração de ambas camadas.

Adicionalmente à importação das tabelas produzidas pelo simulador de nível de enlace físico (MCS, TBS, BLER e SNR), foi necessária a implementação do modelo do canal que determina o nível de atenuação do sinal em visada direta (LOS -Line of Sight) e o desvanecimento, que determina o nível de atenuação adicional devido a reflexões, obstáculos e tempo (NLOS - Non Line of Sight). No caso específico de 5G para áreas remotas, foram necessárias também alterações em parâmetros que caracterizam o uso dos TVWS, a faixa de frequência de transmissão e a coerência com o modelo de canal, a saber:

1. A duração do subframe em 4,6 ms (no LTE padrão é de $1 \mathrm{~ms}$ );

2. A frequência de operação nas faixas de 170 a $450 \mathrm{MHz}$ e 450 a $700 \mathrm{MHz}$, típicas dos canais de TV analógicos e hoje disponíveis nos TVWS;

3. Largura de banda dos blocos TVWS em $24 \mathrm{MHz}$, sendo utilizados 4 canais de 6 $\mathrm{MHz}$ ou 3 canais de $8 \mathrm{MHz}$, conforme os padrões do Brasil e Europa, respectivamente.

4. Quantidade de RBs (Resource Blocks) por grupo: a fim de possibilitar um maior controle pelas camadas superiores, um RBG (Resource Block Group) pode ter apenas $1 \mathrm{RB}$, o que permite uma maior granularidade e flexibilidade para as camadas superiores.

As alterações enumeradas foram realizadas utilizando como base o COLAB [Ferreira et al. 2019]. A Figura 2 apresenta as classes do NS-3 que foram alteradas e que serão descritas a seguir em maiores detalhes.

A estrutura do quadro a ser utilizada no canal do 5G para áreas remotas é diferente da implementada no LENA NS-3. Portanto, o slotframe foi alterado para 4,6ms. Para implementar o novo sub-quadro, uma nova macro chamada SUBFRAME_DURATION foi desenvolvida na classe Lte-Common. Essa alteração implicou mudanças nas classes LteEnbMac, LteEnbPhy, LteEnbMac, LteUePhy, LtePhy, LteSpectrumPhy. Também duas constantes da classe LteEnbPhy foram alteradas: DL_DATA_DURATION e DL_CTRL_DELAY_FROM_SUBFRAME_START. Para estabelecer o controle entre a estação ou UE (User Equipment) e a estação rádio base ou gNB (next Generation Node B), a classe LteEnbRrc que define a camada padrão para o gNB foi atualizada pela macro SUBFRAME_DURATION.

As faixas de frequência de 170 a $450 \mathrm{MHz}$ e 450 a $700 \mathrm{MHz}$, típicas de ambientes rurais ou remotos, foram adicionadas ao módulo LTE por meio da tabela EUTRA [Access 2014], que dispõe de todas as faixas de utilização definidas pelo 3GPP. A alteração da largura de banda afeta o número de blocos de recursos e o cálculo dos RBGs. Esta informação é necessária em várias partes do código do NS-3 e seu cálculo é realizado várias vezes em diferentes estágios. A forma de onda e a modulação descritas na modelagem de canal para áreas remotas não permitem banda de guarda entre os RBs. Portanto, o número de RBs aumenta. Esta tabela está na classe LteSpectrumValueHelper e outras alterações foram necessárias nas classes ComponentCarrier e LteEnbNetDevice. As larguras de banda de $6 \mathrm{MHz}$ e $8 \mathrm{MHz}$ foram adicionadas em diversas partes do código, pois sua utilização é fragmentada, já a utilização de 3 ou 4 canais para formar os $24 \mathrm{MHz}$ é possibilitada através de Carrier Aggregation, na classe ComponentCarrier. As bandas 


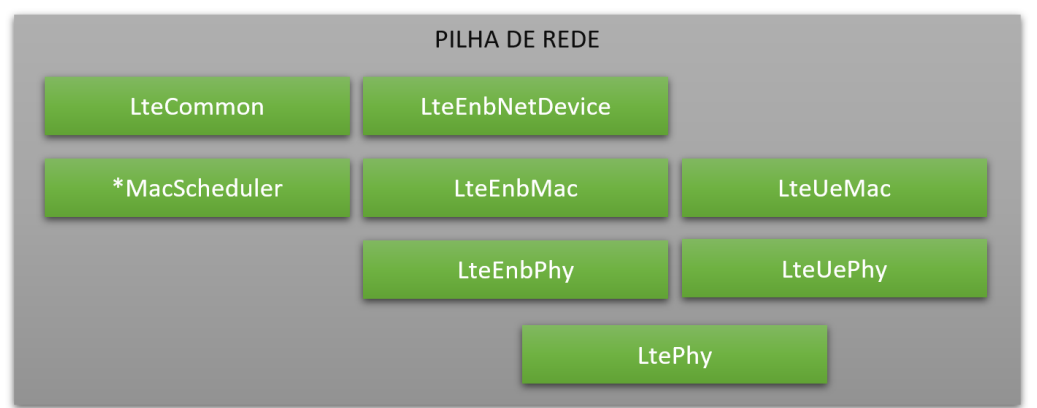

Figura 2. Classes do NS-3 referentes a camada física do 5G-RANGE.

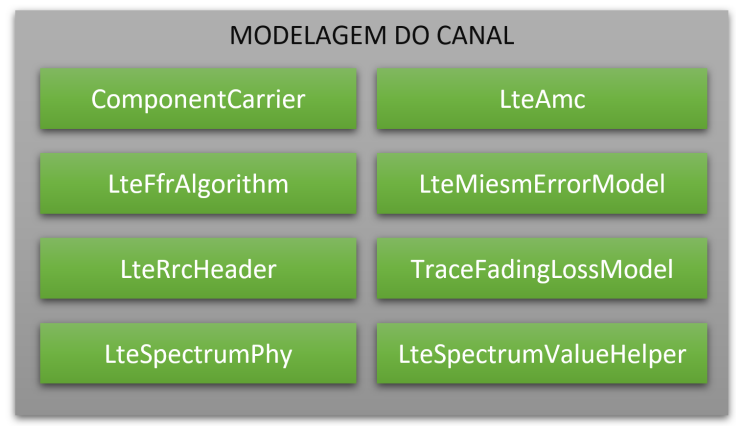

Figura 3. Classes do NS-3 referentes à modelagem do canal.

de $8 \mathrm{MHz}$ e $6 \mathrm{MHz}$ possuem $44 \mathrm{RBs}$ e $33 \mathrm{RBs}$, respectivamente. Com as alterações efetuadas ambas as situações são possíveis usando a agregação de transportadora. No entanto, uma única banda de $24 \mathrm{MHz}$ também foi criada, pois o algoritmo de escalonamento da camada MAC pode ter controle sobre toda a largura de banda disponível.

A especificação padrão do LTE configura uma quantidade mínima de blocos de recursos a serem alocados a um único UE. Nas alterações realizadas foi definido um valor mínimo de $1 \mathrm{RB}$, para aumentar a granularidade do controle do escalonador. Para implementar essa funcionalidade, a classe RbgAllocation no arquivo lte-common.cc que possui o método estático GetRbgSize foi desenvolvido

\subsection{Alterações na Camada de Enlace}

No NS-3, a classe LteSpectrumPhy implementa a função de sensoriamento do canal (Sense), que executa o sensoriamento individual da estação. Para tal, conforme mostrado na Figura 1, o algoritmo de sensoriamento do canal utiliza os parâmetros de SINR e uma curva de probabilidades de detecção de usuário primário, gerados externamente em um simulador no nível de enlace. O algoritmo de sensoriamento implementado (sensingProcedure), verifica a relação SINR de cada um dos RBGs, ou a relação entre o SINR médio dos RBGs, e estima as chances de um PU estar transmitindo baseado na curva de probabilidade. A classe LteEnbPhy foi modificada para encaminhar mensagens de sensoriamento para a camada MAC (Medium Access Control) do gNB associado.

$\mathrm{Na}$ camada de enlace a implementação do sensoriamento colaborativo no NS3 é dispersa em múltiplas classes. Na classe LteSpectrumPhy, no método de recepção (StartRX) foi acrescida uma parte para integração dos dados recebidos e o encaminhamento para uma função de sensoriamento (Sense). A função StartRxDlCtrl 
foi modificada para permitir o recebimento de mensagems de controle com resultados do sensoriamento e o seu encaminhamento para a classe LteEnbPhy. À camada MAC do módulo do UE foi acrescida uma função para montagem da mensagem de controle com resultados do sensoriamento (SendCognitiveMessageC). Na camada MAC do gNB foram acrescidas funções para processar mensagens de sensoriamento das diferentes estações (ReceiveCognitiveMessage) e fazer a fusão dos resultados (mergeSensingReports). Assim também, a função DoSubframeIndication do LTE foi modificada, para implementar a fusão de resultados antes de executar o escalonamento de recursos. Ainda na camada MAC do gNB, a função DoSchedDlTriggerReq dos escalonadores passou a receber um parâmetro adicional, contendo um bitmap informando RBGs ocupados, prevenindo seu uso pelo escalonador.

No COLAB-5 também foi desenvolvido um novo modelo de sensoriamento colaborativo. Na nova versão implementada, que tem como objetivo melhorar a acurácia da detecção e diminuir o número de relatórios das UEs para o $\mathrm{gNB}$, foram acrescidas duas checagens que implementam uma cadeia de Markov de cinco estados. Para cada ciclo de execução, caso o resultado do sensoriamento atual ( $\left.R_{\text {sense }}\right)$ seja equivalente ao do passado $\left(R_{\text {prev }}\right)$, acumula-se a incerteza de eventos idênticos acontecerem consecutivamente $\left(P_{\text {acum }}\right)$. Os resultados do sensoriamento são binários e o acúmulo de probabilidade é feito em saltos de $\frac{1-P_{\text {acum }}}{2}$. Quando esta incerteza $P_{\text {acum }}$ atinge um dado limiar, por exemplo $90 \%$, assume-se que o resultado do sensoriamento $R_{\text {sense }}$ é correto e assim, armazena-se o valor a ser reportado em $R_{\text {markov }}$. Caso o limiar $P_{\text {acum }}$ não tenha sido ultrapassado, o UE não reporta o resultado do sensoriamento atual, e o gNB assume que o resultado reportado no passado continua a ser válido. Essa abordagem apresentou melhores resultados e maior acurácia que os apresentados na versão anterior da ferramenta [Ferreira et al. 2019].

\section{Roteiro de Demonstração}

O código fonte, manual e vídeos estão disponíveis em https: / / gabrielcarvfer. github.io/NS3/COLAB_5G/. Nesta seção, o roteiro de demonstração é ilustrado em duas partes : (1) validação das adaptações no NS-3 na camada física; (2) simulação de um cenário rural, formado por uma célula de $50 \mathrm{~km}$, que demonstra o funcionamento da camada MAC e a alocação oportunística de 04 canais TVWS.

\subsection{Funcionamento da Camada Física}

A validação da camada física no NS-3 foi feita mediante a simulação de um cenário com diferentes números de UEs $(10,20,30,50,100$ e 132). Em todas as simulações, as condições do canal foram consideradas ideais, portanto, nenhuma retransmissão foi realizada. Além disso, foi utilizado o tamanho máximo do bloco, que é de 5888 bits por RB. A cada cenário de validação, diferentes números de UEs enviam pacotes com 736 bytes (a quantidade máxima que se encaixa em um único RB) no intervalo de 4,6 milissegundos para um único ponto. A Figura 4 mostra a proximidade dos resultados simulados (Alcançado) e teóricos (Esperado), além da capacidade máxima do canal (Máximo) baseada em sua modelagem para áreas remotas.

\subsection{Funcionamento da Camada MAC}

Para ilustrar o funcionamento da camada de enlace em termos do sensoriamento colaborativo, usou-se o cenário mostrado na Figura 5(a), que exemplifica uma área rural com 100 


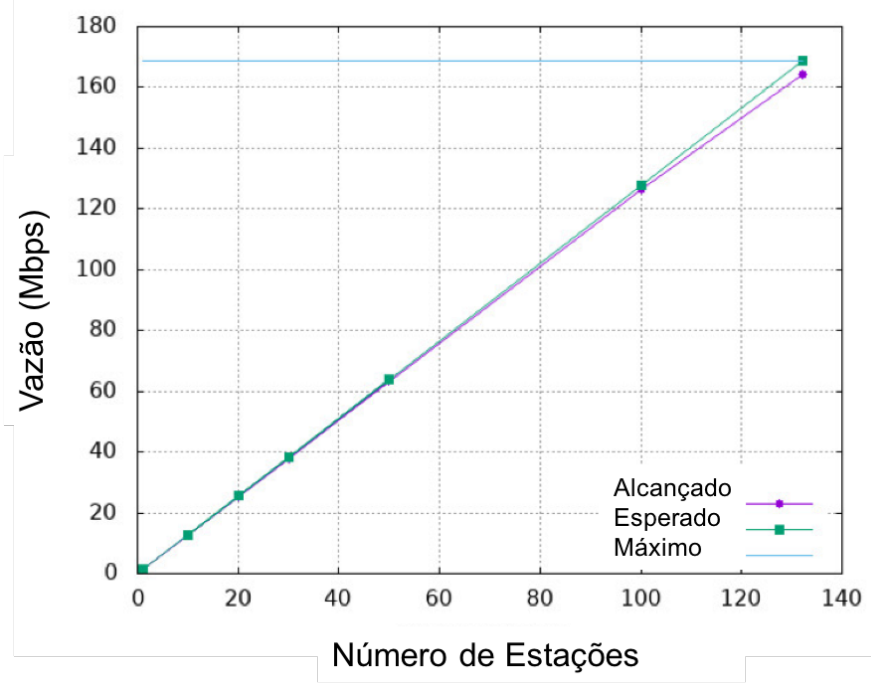

Figura 4. Validação do desempenho após adaptações na Camada Física

UEs pulverizados em uma célula de $50 \mathrm{~km}$ de raio, centrada no eNB. São utilizados quatro canais TVWS, ocupados aleatoriamente por um PU associado ao canal. Os parâmetros de potência de transmissão, ganho de antena e modelagem do canal são especificados em [5G-Range 2018]. As Figuras 5(b) e 5(c) ilustram os resultados do sensoriamento colaborativo utilizando a camada física do COLAB-5. A técnica de fusão proposta é comparada com outras técnicas clássicas k-de-n, apresentando redução na fração de quadros reportados, reduzindo tráfego de controle. São apresentados também acurácia, falsos positivos (causam inutilização do espectro quando utilizado com acesso dinâmico ao espectro) e falsos negativos (podem causar colisões com o usuário primário do canal).

\section{Conclusão e Trabalhos Futuros}

O COLAB-5 é uma ferramenta resultante da adaptação da pilha LTE do NS-3 para incluir funcionalidades de uma rede $5 \mathrm{G}$ rural referentes à camada física e camada de enlace com sensoriamento colaborativo. A implementação procura reduzir a complexidade das simulações no NS-3 e permite definir diversos parâmetros necessários para implementar diferentes cenários de redes $5 \mathrm{G}$. Como trabalhos futuros, pretende-se implementar um novo escalonador da camada MAC e integração da com a ferramenta geradora de tráfego sintético EROS-5.

\section{Agradecimentos}

Este trabalho foi realizado com suporte do projeto 5G-Range (www.5g-range.eu), aprovado na 4a. Chamada EU-BR em TICs, financiada pelo CTIC/RNP/MCTIC.

\section{Referências}

5G-Range (2018). Spectrum sensing to complement databases. Technical report. http://5g-range.eu/wp-content/uploads/2018/04/D4 . 2-Spectrum-Sensing-to-Complement-Databases.pdf.

Access, E. U. T. R. (2014). User equipment (ue) radio transmission and reception. Technical report, ETSI. 


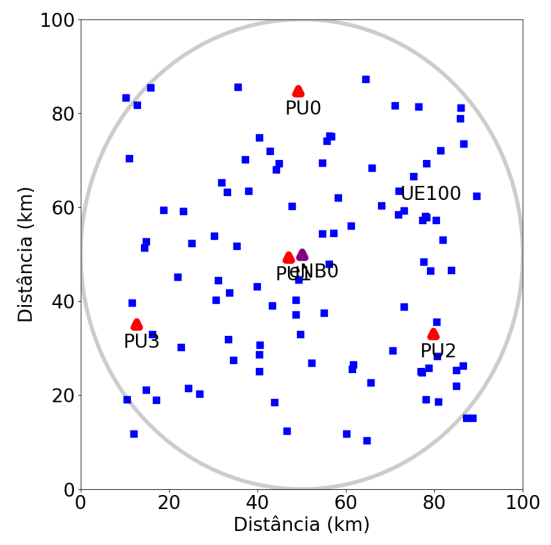

(a) Topologia

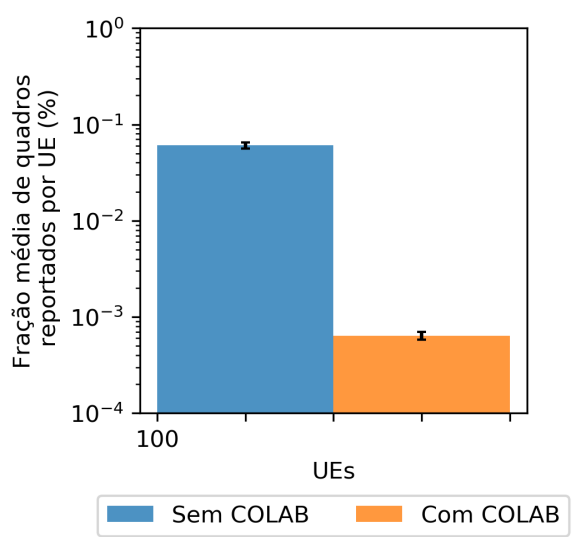

(b) Fração de quadros reportados por UE

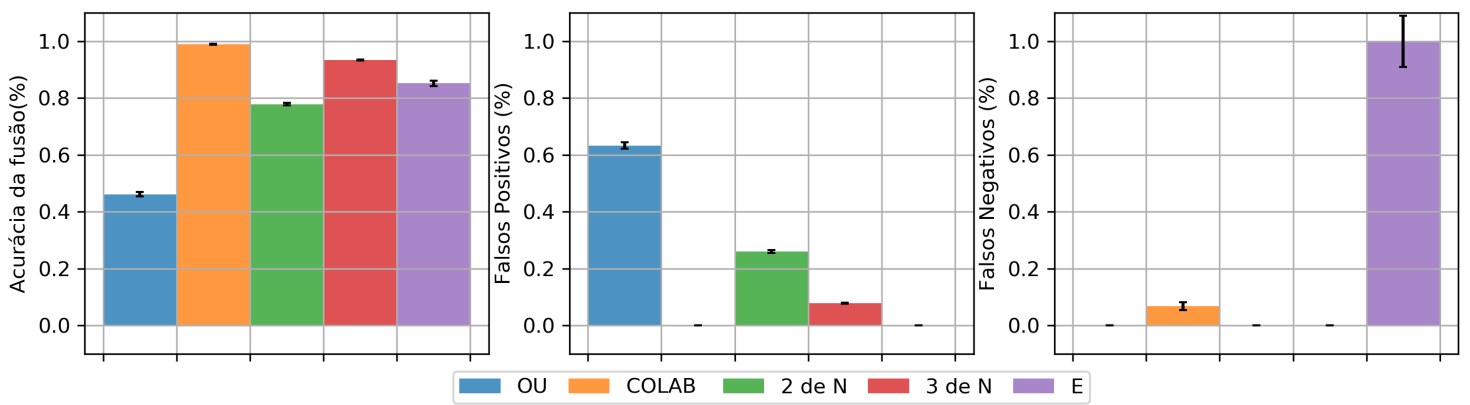

(c) Desempenho de diferentes algoritmos de fusão para sensoriamento colaborativo no cenário com 100 UEs

Figura 5. Cenário de simulação e resultados

Agnelli, S., Feltz, P., Griffiths, P., and Roth, D. (2014). Satellite's role in the penetration of broadband connectivity within the european union. In 2014 7th Advanced Satellite Multimedia Systems Conference and the 13th Signal Processing for Space Communications Workshop (ASMS/SPSC), pages 306-311.

Association, G. (2017). The mobile economy. latin america the caribbean. Technical report.

CTTC (2019). The first release of 5g-lena is available. http://www.cttc.es / the-first-release-of-5g-lena-is-available/.

Ferreira, G., Barreto, P. S., Caetano, M., Alchieri, E., Vartiainen, J., Karvonen, H., Matinmikko-Blue, M., and Seki, J. (2019). A tool for developing collaborative sensing and cognitive mac layer solutions for $5 \mathrm{~g}$ in rural areas. In 6th International Symposium on Wireless Communication Systems (ISWCS). IEEE.

NSNAM (2019). ns-3 network simulator. https : / / www . nsnam. org/.

Sultan, A. (2019). 21.915 release 15. Technical report. https://portal.3gpp. org/desktopmodules/Specifications/SpecificationDetails. aspx?specification $I d=3389$.

Yau, K.-L. A., Qadir, J., Wu, C., Imran, M. A., and Ling, M. H. (2018). CognitionInspired 5G Cellular Networks: A Review and the Road Ahead. IEEE Access, 6:35072 -35090 . 